\title{
Aplicación de Principios de Nutrición de Ganado Lechero en la Producción de Leche y Carne con Ingredientes Tradicionales y Alternativos
}

\author{
Hugo A. Ramírez Ramírez ${ }^{1}$
}

Resumen. El aumento esperado en la población mundial tiene implicaciones de alto impacto en la producción animal porque se requerirá producir más alimentos de origen animal con baja disponibilidad de recursos naturales. Nuestro conocimiento y capacidad para desarrollar tecnologías que permitan la utilización eficiente de los recursos alimenticios para la producción de alimentos serán elementos críticos para el sustento de la población. Como parte de los avances tecnológicos en la alimentación animal, podemos evaluar recursos forrajeros que incrementen la producción, mejoren la eficiencia o una combinación de ambos. Actualmente se cuenta con híbridos de maíz y otras especies con características ventajosas para la producción de forraje que permiten una mejor digestibilidad y producción animal. Además, el desarrollo de varios procesos agroindustriales para la producción de satisfactores humanos conlleva a la creación de subproductos que se pueden utilizar en la producción de carne y leche de bovinos. Ejemplo de esto es la producción de etanol y biodiesel con la consecuente producción de granos de destilería y glicerol para la alimentación animal. Otros avances tecnológicos en la nutrición animal se pueden ver en el desarrollo de modelos computacionales para la formulación de dietas y tecnología aplicada a nutrientes específicos como amino ácidos protegidos. En este artículo se presenta información actual sobre nutrición y estrategias de alimentación de ganado lechero y de carne utilizando ingredientes tradicionales, subproductos industriales y suplementos nutritivos que permiten mejorar la producción pecuaria ya sea en términos de rendimiento, costo de alimentación o eficiencia alimenticia.

Palabras clave: Alimentos de subproductos, forrajes, nutrición de rumiantes.

\section{Application of Dairy Nutrition Principles for Milk and Meat Production Using Traditional and Alternative Ingredients}

\begin{abstract}
The expected increase in the world's population has high-impact implications regarding animal production because more food of animal origin will need to be produced with an ever-shrinking availability of natural resources. Our knowledge and capacity to develop technologies that allow efficient utilization of animal feed resources for human food production will play a critical to sustain our future livelihood. As part of the technological advances in animal feed, we can evaluate forage resources to increase production, improve animal efficiency or a combination of both. Currently there are corn hybrid and other crops with favorable characteristics for forage production that allow for improved digestibility and animal performance. In addition, the development of different agro-industrial processes leads to the creation of byproducts that can be used in the production of meat and milk from cattle. An example of this is the production of ethanol and biodiesel with the subsequent production of distiller's grains and glycerol for animal feed. Other technological advances in animal nutrition can be seen in the development of computer models for diet formulation, and technology applied to specific nutrients such as protected amino acids. This article presents current information on nutrition and feeding strategies for dairy and beef cattle using traditional ingredients, industrial byproducts and nutritional supplements that improve livestock production either in terms of yield, cost of production, or feed efficiency.
\end{abstract}

Keywords: Byproduct feeds, forages, ruminant nutrition.

\footnotetext{
${ }^{1}$ Animal Science Department. lowa State University. 806 Stange Road. Kildee Hall 313. Ames, lowa, Estados Unidos, 50011-1178. Correo electrónico hramirez@iastate.edu
} 


\section{Introducción}

Se estima que la población mundial rebase los 9,000 millones para el año 2050 (United Nations 2013); este crecimiento demográfico acarrea una situación difícil de balancear e imposible de evitar: producir más alimentos con una base de recursos naturales que continúa en declive. Es por ello que en los próximos años será necesario aplicar y refinar nuestro conocimiento referente a la nutrición y alimentación del ganado para incrementar rendimiento y utilizar los recursos alimenticios para mejorar la eficiencia productiva.

La producción de leche se basa en el aprovechamiento de la capacidad que tienen los rumiantes para digerir celulosa y hemicelulosa, y convertir proteína de baja calidad en proteína con alto valor biológico para el consumo humano. Además de esta habilidad natural, la selección genética para incrementar el rendimiento productivo ha permitido que los animales actuales tengan una alta capacidad de producción. Es por esta razón, que el entendimiento de los procesos digestivos y metabólicos es una herramienta básica para desarrollar estrategias y programas de alimentación que permita a los animales expresar su potencial genético. Por ejemplo, el progreso en la selección genética y en la nutrición del ganado lechero han logrado que la producción promedio anual de leche en Estados Unidos sea 14\% superior comparada con la década anterior; estas cifras corresponden a 10,100 kg/vaca en 2014 mientras que en el año 2005 se registraron 8,850 $\mathrm{kg} / \mathrm{vaca}$ (USDA-NASS 2016). Es evidente que estos animales tienen altos requerimientos nutricionales y nuestra labor como zootecnistas profesionales debe ser la de formular dietas que permitan alcanzar altos niveles productivos al menor costo sin dejar de lado la salud animal y las implicaciones ambientales.

\section{Reseña del Refinamiento en la Nutrición de Rumiantes}

La crianza y aprovechamiento del ganado lechero ha pasado por una serie de cambios evolutivos desde la ganadería de trashumancia hasta los sistemas de estabulación actuales. Los métodos de alimentación y nutrición también han ido evolucionando a través del tiempo. Ejemplo de esto es el desarrollo del sistema de fibra detergente neutro (FDN) y ácido (FDA) y sus implicaciones en la nutrición de rumiantes (Van Soest et al. 1991). La proporción de FDN de un forraje o de una dieta se relaciona la capacidad de ingesta de alimento de tal modo que a mayor contenido de FDN menor capacidad de consumo debido al llenado del rumen. Por otro lado, el contenido de FDA tiene una relación inversa con la digestibilidad de un alimento.

Otro aspecto que ha evolucionado en términos de nutrición del ganado lechero es el desarrollo de la partición de la proteína basada en la degradación ruminal. Este sistema considera proteína degradable en rumen (PDR), proteína no degradable en rumen (PNDR) y proteína metabolizable (PM). La PDR es aquella que se degrada en el rumen por acción microbiana para la síntesis de proteína microbiana. La PNDR pasa del rumen al abomaso e intestino delgado junto con la proteína microbiana y se combina con secreciones internas denominadas proteína endógena y para formar PM (NRC 2001).

En términos de energía, contamos con un sistema que considera las pérdidas energéticas en la heces para determinar energía digestible; pérdidas de energía en orina y gases resultan en la estimación de energía metabolizable; y finalmente el descuento energético debido al incremento térmico permite cuantificar la energía neta que el animal puede utilizar (NRC 1996). Las tendencias actuales señalan que las formulaciones de dietas para bovinos serán cada vez más sofisticadas con el uso de modelos matemáticos más precisos para predecir el desempeño animal y se considerarán otros factores para refinar la alimentación del ganado. La incorporación de aminoácidos protegidos en las dietas, nitrógeno noproteico de liberación retardada, probióticos y mejoradores de la fermentación ruminal y atención al perfil de ácidos grasos de la dieta, son ejemplos de las nuevas tecnologías para la alimentación del ganado de carne y leche.

\section{Utilización de Ensilado de Maíz en la Alimentación del Ganado Lechero}

La alimentación del ganado bovino se basa en la combinación de forrajes y concentrados para proveer los nutrientes necesarios para cubrir los requerimientos de mantenimiento y producción. El ensilado de maíz y maíz en grano se han utilizado para aportar energía en la dieta y aún permanecen como objeto de estudio para incrementar la utilización de los nutrientes contenidos en estos alimentos. El ensilado del maíz es la base de las dietas para ganado lechero 
en Estados Unidos (Akay y Jackson, 2001) y otras partes del mundo, por eso es importante explorar estrategias para obtener mayor eficiencia nutritiva. Unas estrategias para mejorar el aprovechamiento del ensilado de maíz incluyen modificaciones del procesamiento de cosecha (Cooke y Bernard, 2005; Vanderwerff et al. 2015) y la selección de híbridos (Akay y Jackson 2001; Ramirez-Ramirez et al. 2012).

\section{Selección de Híbridos}

Entre los híbridos de maíz que se han desarrollado para mejorar la composición nutritiva del grano y ensilado se incluyen aquellos con la característica Nutridense (NDS), los de grano ceroso y el maíz forrajero de nervadura café. Los híbridos de maíz con composición mejorada del grano que contienen la característica NDS cobran valor cuando el consumo de energía es limitado, por ejemplo, para las vacas en periodo de transición. El maíz tipo NDS tiene un germen de mayor tamaño y por ende contienen más aceite y provee más energía; además el contenido de proteína también es mayor comparado con el maíz convencional (Akay y Jackson 2001; Benefield et al. 2006). Los resultados con este tipo de maíz varían dependiendo del esquema de alimentación ya que en unos estudios se utiliza ensilado de híbridos DNS y en otras ocasiones solo el grano DNS. Akay y Jackson (2001) compararon el desempeño productivo de vacas alimentadas con ensilado de maíz DNS y un híbrido convencional y reportaron un consumo de materia seca similar con un incremento en la producción de leche corregida por energía. Como consecuencia de esto, también se observó un incremento en la eficiencia alimenticia siendo $1.38 \mathrm{~kg}$ leche/ $\mathrm{kg}$ MS para el híbrido convencional y $1.43 \mathrm{~kg}$ leche $/ \mathrm{kg}$ MS.

Los híbridos de nervadura café tienen una mutación genética denominada bm3; esta mutación ocurre naturalmente y produce plantas con bajo contenido de lignina. El bajo contenido de lignina resulta en plantas menos rígidas y con mayor potencial de digestibilidad (Barrière y Argillier 1993). La mayoría de los estudios con ganado lechero han reportado un incremento promedio de $1 \mathrm{~kg} /$ día en el consumo de materia seca, superioridad de $4 \%$ en la digestibilidad de FDN y aumento de $1.5 \mathrm{~kg} /$ día en la producción de leche (Ferraretto y Shaver 2015). La elección de híbridos de nervadura café es una decisión firme para la producción de forraje sin flexibilidad de cosechar para grano. Esto se debe a que el rendimiento de toneladas de forraje por hectárea es menor comparado con híbridos de doble propósito (Barrière y Argillier 1993). Akins y Shaver (2014) reportaron una diferencia de 13 toneladas de forraje fresco equivalente a 6 toneladas de materia seca entre el maíz de doble propósito y el maíz de nervadura café. Esta desventaja aparente se puede ver compensada si se analiza la producción total de leche relativa a las toneladas de forraje cosechadas, es decir kilogramo de leche por tonelada de forraje. Para esta evaluación se puede consultar el programa Milk2006 Corn Silage desarrollado en la Universidad de Wisconsin-Madison y disponible en esta dirección: http://shaverlab.dysci.wisc.edu/spreadsheets/

El grano de maíz es una fuente rica de energía porque contiene aceite y almidón. El tipo de maíz mejorado tipo ceroso contiene $100 \%$ amilopectina, un tipo de almidón ramificado, comparado con el maíz convencional que contiene $75 \%$ de amilopectina y $25 \%$ amilosa, un tipo de almidón de cadena lineal (Akay y Jackson 2001). La potencial ventaja de este tipo de almidón ramificado es que posee múltiples sitios de adherencia para las bacterias ruminales; esto puede resultar en una mejora en la digestibilidad del almidón y consiguientemente mayor producción de leche y mejor eficiencia alimenticia (Akay y Jackson 2001).

\section{Modificaciones del Proceso de Cosecha}

El momento y el método de cosecha de forrajes son factores de suma importancia que tienen impacto sobre el rendimiento en campo y la calidad del forraje. El maíz destinado para ensilado comúnmente se cosecha cuando la planta tiene un contenido de humedad cercano al $65 \%$, equivalente a $35 \%$ de materia seca. Procesos para incrementar la calidad del forraje al momento de cosecha incluyen el procesamiento del grano y recientemente un método de cosecha llamado Shredlage, este término no tiene traducción directa, pero una aproximación sería desgarre.

El procesamiento del grano se refiere a la cosecha del maíz con una cosechadora autopropulsada a la cual se le ha adaptado un procesador de rodillos para fracturar o triturar los granos de maíz para romper el pericapio. Al romper el pericarpio se expone el almidón contenido en el grano de maíz con el objetivo de incrementar la fermentación ruminal y la digestibilidad de la dieta para incrementar la producción de leche. Ebling y Kung (2004) indicaron un incremento de 1.8 $\mathrm{kg} / \mathrm{día}$ en la producción de leche entre una dieta con 
Ramírez Ramírez: Aplicación de Principios de Nutrición de Ganado Lechero en la Producción de Leche y Carne

ensilado procesado y otra con ensilado obtenido por cosecha convencional. Esta observación es paralela a un incremento de aproximadamente $10 \%$ en la digestibilidad de la materia seca y materia orgánica. En cuestiones de digestibilidad de FDN es común observar aumentos de $10 \%$ con ensilado procesado (Ebling y Kung 2004; Cooke y Bernard 2005). En otro experimento, Weiss y Wyatt (2000) reportaron que vacas que consumieron el ensilado convencional digirieron $94 \%$ del almidón consumido mientras que vacas que consumieron el ensilado procesado digirieron 97\%; Ebling y Kung (2004) reportaron una ventaja de $10.2 \%$ en la digestibilidad del almidón asociada con una reducción en la excreción de granos enteros de maíz. Contenido de granos enteros de maíz por cada $250 \mathrm{~g}$ de heces fue de 20 granos en las vacas que consumieron ensilado sin procesar mientras que las vacas que consumieron ensilado procesado excretaron 3 granos enteros por cada $250 \mathrm{~g}$ de heces. Es evidente que la aplicación de los rodillos para procesar granos mejora las características nutritivas del ensilado de maíz. Para obtener mejores resultados se recomienda que los rodillos se calibren a una separación de $2 \mathrm{~mm}$ y revisar periódicamente que los granos salgan fracturados o totalmente fragmentados al momento de la cosecha. Los granos que están simplemente aplastados no tendrán el mismo efecto positivo ya que el pericarpio puede permanecer relativamente intacto si los rodillos tienen una separación mayor.

La cosecha del maíz para producir Shredlage es con una cosechadora autopropulsada a la cual se le ha instalado un procesador con dos rodillos de superficie cuadriculada, que rotan a distinta velocidad y provocan mayor desgarre de las fibras de la planta y fracturación de los granos. Este tipo de cosecha requiere que la longitud teorética de corte sea de entre 26 y $30 \mathrm{~mm}$, la cual es ligeramente más larga que el ensilado convencional. Esta tecnología está ganando popularidad y cada vez hay más interés en estudiar las formas de sacarle el mayor provecho. La teoría detrás de este procesamiento se basa en que el desgarre del material vegetal incrementa la superficie de contacto y provee mayor cantidad de sitios para que las bacterias del rumen colonicen y digieran el material de forma más extensa. El incremento en longitud de corte no parece afectar la capacidad de consumo de materia seca. Vanderwerff et al. (2015) reportaron que las vacas consumiendo una dieta con Shredlage consumieron una cantidad similar de materia seca e invirtieron igual tiempo rumiando, comparado con las vacas que consumieron el ensilaje procesado. En el trabajo de Ferraretto y Shaver y en el de Vanderwerff et al. (2015) se reportaron diferencias en la producción semanal de leche, con ventaja al Shredlage, pero quizás lo más interesante es que las vacas que consumieron las dietas de prueba mostraron una persistencia notable.

\section{Utilización de Subproductos Agroindustriales en la Producción de Carne}

La disponibilidad de subproducto varía mucho dependiendo de los cultivos que se produzcan en la zona cercana al corral de engorda. Por ello, es importante reconocer cuáles recursos se encuentran cercanos a las zonas ganaderas y aprovechar la oportunidad de incluir productos con valor nutricional y bajo precio. A continuación se presentan ejemplos de subproductos de importancia para la producción de carne de res en Estados Unidos y Brasil.

En la zona maicera de Estados Unidos se produce la mayoría del etanol para combustible a partir de la fermentación del maíz; este proceso solamente utiliza la fracción de almidón del grano de maíz. El almidón es aproximadamente el $30 \%$ del grano, por lo tanto, después de la fermentación y destilación, los componentes restantes se concentran al triple en los subproductos, comúnmente llamado granos de destilería o granos destilados (GD). En promedio, el contenido del extracto etéreo y de la FDN es de 12 y $38 \%$, respectivamente. Estos valores muestran el valor energético de los GD para los rumiantes. Además, el contenido promedio de proteína cruda (PC) de GD es $30 \%$ y la mayoría de los informes sobre el contenido PNDR indican que es alrededor de 50 a $55 \%$ de la PC, pero oscila entre 33 y 63\% (NRC 2001; Castillo-López et al. 2013). La inclusión de $20 \%$ de GD en las dietas de engorde intensivo se ha traducido en una mayor rentabilidad económica (Buckner et al. 2008). En una revisión extensiva, Klopfenstein et al. (2008) indicaron que novillos de engorda que consumen hasta $40 \%$ de GD tienen ganancias de peso mayores a los novillos que consumen dietas basadas en maíz, la ventaja es en promedio 160 g/día (1.73 vs 1.57 kg/día).

Otro subproducto industrial que se está explorando es el glicerol derivado de la industria del biodiesel. Este subproducto puede reemplazar al maíz como fuente de energía y es altamente fermentable en el rumen (Donkin 2008). En un estudio con ganado Nellore, van Cleef (2014) reportó que la mayor 
ganancia diaria de peso fue con $15 \%$ de glicerol en la dieta como reemplazo del maíz.

En países tropicales pude haber más variedad de subproductos con valor nutritivo para el ganado derivados del procesamiento de frutas, el siguiente cuadro presenta el valor nutritivo de algunos de estos productos (Cuadro 1).

Cuadro 1. Valor nutritivo de subproductos tropicales para la alimentación de bovinos. Fuente: Feedstuffs - 2014 Reference issue \& buyers guide.

\begin{tabular}{|c|c|c|c|c|c|c|c|c|c|c|c|c|c|c|}
\hline \multirow[b]{2}{*}{ Subproducto } & \multicolumn{2}{|c|}{$\%$} & \multicolumn{3}{|c|}{ Mcal/kg } & & \multicolumn{8}{|c|}{$\%$} \\
\hline & $\mathrm{MS}^{\S}$ & $\mathrm{NDT}^{£}$ & $\mathrm{ENI}^{\circ}$ & $\mathrm{ENm}^{\text {F }}$ & $\mathrm{ENg}^{\mathrm{F}}$ & $\mathrm{PC}^{*}$ & $E E^{\|}$ & $\mathrm{FC}^{\mathrm{U}}$ & $\mathrm{FDA}^{€}$ & $\begin{array}{l}\text { Ceni- } \\
\text { zas }\end{array}$ & $\mathrm{Ca}$ & $\mathrm{P}$ & $\mathrm{K}$ & $\mathrm{Mg}$ \\
\hline Pasta de coco & 92.0 & 82.0 & 1.90 & 2.00 & 1.34 & 22.4 & 6.9 & 12.8 & 19.0 & 7.3 & 0.22 & 0.66 & 1.62 & 0.33 \\
\hline Piña & 14.7 & 80.1 & 1.85 & 1.90 & 1.21 & 2.7 & 1.4 & 2.7 & 3.0 & 2.7 & 0.14 & 0.07 & - & - \\
\hline $\begin{array}{l}\text { Pasta de } \\
\text { aguacate }\end{array}$ & 91.0 & 50.0 & 1.10 & 1.01 & 0.28 & 20.3 & 1.2 & 19.3 & 24.0 & 12.4 & - & - & - & - \\
\hline Bananas & 24.3 & 84.1 & 1.94 & 2.02 & 1.34 & 4.5 & 0.8 & 2.1 & 3.0 & 3.3 & 0.03 & 0.11 & - & - \\
\hline $\begin{array}{l}\S \text { MS, materia } \\
£ \text { NDT, nutrie } \\
\& \text { ENI, } \\
{ }^{\ddagger} \text { ENm, } \\
\text { ENg, }\end{array}$ & $\begin{array}{l}\text { eca } \\
\text { es di }\end{array}$ & stible & les & & & & $\begin{array}{l}\text { PC, } \\
\text { Extra } \\
\text { Fibra } \\
=i b r a\end{array}$ & $\begin{array}{l}\text { eina } \\
\text { etér } \\
\text { uda } \\
\text { terge }\end{array}$ & e ácido & & & & & \\
\hline
\end{tabular}

\section{Perspectivas para la Ganadería Tropical}

El clima tropical ofrece ventajas para la producción de forrajes y es importante aprovechar los recursos naturales con los que se cuenta. Probablemente la mayor desventaja es la tasa de crecimiento y maduración del forraje durante la temporada húmeda porque se pueden encontrar situaciones en las que la producción de forraje excede el consumo y al llegar la temporada seca el forraje ha perdido calidad nutritiva debido al avance de la maduración. En este tipo de situaciones es conveniente desarrollar la cultura de estimación de presupuestos forrajeros e implementar prácticas de conservación de forrajes como ensilaje y henilaje. Estas prácticas permiten preservar el valor nutritivo del forraje al momento de corte para mantener un suministro nutricional relativamente uniforme durante la temporada en la que el forraje en pie no es tan abundante.

El trópico ofrece una abundancia de frutos con alto contenido de azúcares que se pueden utilizar en la alimentación de bovinos. Es importante ubicar que subproductos están disponibles en la región, considerar el valor nutritivo, el costo del producto incluyendo transporte. Estos alimentos son propensos a la fermentación y descomposición, por lo tanto se debe considerar la tasa de consumo para qué cantidad de alimento se necesita y con qué rapidez se puede consumir. Si hay un excedente de alimento se pueden explorar formas de conservación similares a las del ensilado; la clave de la conservación de recursos alimenticios fermentados radica en evitar la presencia de oxígeno.

\section{Visión sobre los Futuros Avances en la Nutrición de Rumiantes}

La nutrición de rumiantes aprovecha la simbiosis entre el animal huésped y los microorganismos que viven en el rumen. La mayoría de los esfuerzos actuales se enfocan en el entendimiento, manipulación y modulación de los procesos digestivos y metabólicos del animal huésped. Sin embargo, los avances en la biotecnología permiten estudiar ecosistemas complejos como el rumen y esfuerzos recientes y futuros se enfocan en la modulación del microbioma ruminal para promover el desarrollo poblaciones de bacterias eficientes, suprimir las emisiones de metano y establecer vínculos entre los procesos metabólicos de las bacterias y los efectos en el metabolismo del huésped. Las tendencias actuales indican que se fortalecerá la interacción entre la nutrición de rumiantes clásica y las ciencias ómicas para lograr avances en el conocimiento y desarrollar estrategias para mantener el progreso en la producción animal y poder brindar sustento a la población global. 


\section{Conclusiones}

El interés en el aprovechamiento de los recursos forrajeros y recursos alimenticios alternativos ha renacido a raíz de las condiciones de mercado y la volatilidad en los precios de los granos. Esta situación nos motiva a seguir explorando maneras para incrementar la utilización de nutrientes en forrajes y subproductos agroindustriales que favorezcan la producción de leche y carne. La adopción en implementación de tecnología como híbridos mejorados, métodos de cosecha y conservación, y utilización de suplementos alimenticios cobran más importancia en vísperas del incremento en la demanda de productos de origen animal y la escasez de recursos naturales. Así mismo, se continuará con los esfuerzos para reducir el impacto ambiental de la ganadería a través de la formulación de dietas que reduzcan las emisiones de metano y la excreción de nutrientes para incrementar la eficiencia alimenticia.

\section{Literatura Citada}

Akay, V. y J A. Jackson Jr. 2001. Effects of Nutridense and waxy corn hybrids on the rumen fermentation, digestibility and lactational performance of dairy cows. Journal of Dairy Science 84:1698-1706.

Akins, M.S. y R.D. Shaver. 2014. Influence of corn silage hybrid type on lactation performance by Holstein dairy cows. Journal of Dairy Science 97:7811-7820.

Barrière, Y. y O. Argillier. 1993. Brown-midrib genes of maize: a review. Agronomie 13:865-876.

Benefield, B.C., M. Liñeiro, I.R. Ipharraguerre y J.H. Clark. 2006. Nutridense corn grain and corn silage for dairy cows. Journal of Dairy Science 89:1571-1579

Buckner, C.D, T.L. Mader, G.E. Erickson, S.L. Colgan, D.R. Mark, V.R. Bremer, K.K. Karges y M.L. Gibson. 2008. Evaluation of dry distillers grains plus solubles inclusion on performance and economics of finishing beef steers. The Professional Animal Scientist 24:404-410

Castillo-Lopez, E., T.J. Klopfenstein, S.C. Fernando y P.J. Kononoff. 2013b. In vivo determination of rumen undegradable protein of dried distillers grains with solubles and evaluation of duodenal microbial crude protein flow. Journal of Animal Science 91:924-934

Cooke, K.M. y J.K. Bernard. 2005. Effect of length of cut and kernel processing on use of corn silage by lactating dairy cows. Journal of Dairy Science 88:310-316

Donkin, S.S. 2008. Glycerol from biodiesel production: the new corn for dairy cattle. Revista Brasileira de Zootecnia. 37 suplemento especial: 280-286
Ebling, T.L. y L. Kung. 2004. A comparison of processed conventional corn silage to unprocessed and processed brown midrib corn silage on intake, digestion, and milk production by dairy cows. Journal of Dairy Science 87:2519-2526

Ferraretto, L.F. y R.D. Shaver. 2012. Effect of corn shredlage on lactation performance and total tract starch digestibility by dairy cows. The Professional Animal Scientist 28:639-647

Ferraretto, L.F. y R.D. Shaver. 2015. Effects of whole-plant corn silage hybrid type on intake, digestion, ruminal fermentation, and lactation performance by dairy cows through a meta-analysis. Journal of Dairy Science 98:2662-2675

National Research Council. 1996. Nutrient requirements of beef cattle. 7th rev.Ed. National Academy of Sciences. Washington, DC.

National Research Council. 2001. Nutrient requirements of dairy cattle. 7th rev.Ed. National Academy of Sciences. Washington, DC

Ramirez Ramirez, H.A., K. Nestor, L.O. Tedeschi, T.R. Callaway, S.E. Dowd, S.C. Fernando y P.J. Kononoff. 2012. The effect of brown midrib corn silage and dried distillers' grains with solubles on milk production nitrogen utilization, and microbial community structure in dairy cows. Canadian Journal of Animal Science 92:365-380.

United Nations. 2013. World population projected to reach 9.6 billion by 2050 . En línea: https://www.un.org/development/desa/en/news/populatio n/un-report-world-population-projected-to-reach-9-6billion-by-2050.html con acceso el 27 de marzo, 2016

United States Department of Agriculture - National Agriculture Statistics Service. 2016. Milk: production per cow by year, US. En línea: http://www.nass.usda.gov/Charts_and_Maps/Milk_Prod uction_and_Milk_Cows/cowrates.php Con acceso el 26 de marzo, 2016

van Cleef, E.H. C.B., J.M. Bertocco Ezequiel, A.P. D`Aurea, V. Ruiz Fávaro y J. Borsari Dourado Sancanari. 2014. Crude glycerin in diets for feedlot Nellore cattle. Revista Brasileira de Zootecnia 3:86-91

Vanderwerff, L.M., L.F. Ferraretto y R.D. Shaver. 2015. Brown midrib corn shredlage in diets for high producing dairy cows. Journal of Dairy Science 98:5642-5652

Van Soest, P.J., J.B. Robertson y B.A. Lewis. 1991. Methods for dietary fiber, neutral detergent fiber, and nonstarch polysaccharides in relation to animal nutrition. Journal of Dairy Science 74:3583-3597

Weiss, W.P. y D.J Wyatt. 2000. Effect of oil content and kernel processing of corn silage on digestibility and milk production by dairy cows. Journal of Dairy Science 83:351-358.

Recibido para publicación el 1 de abril de 2016.

Aceptado para publicación el 16 de julio de 2016. 